\title{
Appendix I.I
}

\section{US consular officers in Ireland, 1790-1913}

Belfast (consulate)

James Holmes

William Phelps

1796-1815

James Luke

1815

Samuel Luke

1815-20

Thomas W. Gilpin

1820-30

James Shaw

1830-42

Thomas W. Gilpin

1842-5

Thomas McClure

1845-7

James McDowell

1848

John C. O’Neill

1848-9

John Higgins

1853-4

James McAdam

1854-8

James J. Higgins

1854

James Arrott

1856,1858

Theodore Frean

1858

John Young

1859-61

Gwynne Harris Heap

1861-6

Thomas K. King

1866, 1867

James M. Donnan

1867-9

Lewis Richmond

1873-80

Arthur B. Wood

1880-1

1881-5

George W Savage

1885-9

Samuel Ruby

1889-93

James B. Taney

Malcolm T. Brice

1893-7

Louis Mantell

1895 December

1897 (deputy)

W. W. Touvelle

1897-1902

Edward Harvey

Samuel S. Knabenshue

1902-4 (deputy)

Hunter Sharp

1905-9

1914-20

Vice consuls

John M. Savage

Claude T Ruby

1885-9

1895 
Malcolm T. Brice 1897-8

Arthur R. Touvelle $\quad 1904$

Edward Harvey 1906

Hugh H. Watson 1914

Cork/Cove/Cobh/Queenstown (consulate)

John Church

$1797-1815$

Michael Hogan

$1815-17$

Jacob Mark

1817-26

Reuben Harvey

$1827-36$

John Murphy

1836-49

Alfred Mitchell

1849-53

John Higgins

1853-4

Hugh Keenan

1854-9

Robert Dowling

1859-61

Patrick J. Devine

1861-2

Edwin G. Eastman

$1862-9$

E. D. Neill

William H. Townsend

1869-70 (acting consul)

Lewis Richmond

1870-5

Edward P. Brooks

1875-80

1880-2

George Dawson

John J. Piatt

1881 (acting)

1882-93

Henry J. Kress

1893

Lucien J. Walker

1893-7

Daniel Swiney

1897-1905

Edwin N. Gunsaulus

1905-6

George E. Chamberlin

1910-14

Vice consuls

Nicholas George Seymour

George Dawson

Robert Seymour, James William Scott

1834-49

1871-80 (acting, deputy and vice)

1886-92

1889

Arthur Donn Piatt

1892

James W. Scott

1897 
Dublin (consulate)

William Knox

Joseph Wilson

Thomas English

Isaac English

Thomas Wilson

Hugh Keenan

James Foy

M. L. Lynch

Michael Lynch

Robert L. Longhead

Hugh Keenan

James Arrott

Michael Lynch

Samuel W. Talbott

Henry B. Hammond

Edward Neill

Wilson King

Benjamin H. Barrows

J. L. McCaskill

Alexander J. Reid

Wilbur Allan Reid

John J. Piatt

Newton B. Ashby

Joshua Wilbour

Rufus Waterman,

Arthur K. Moe

Edward Adams

Vice consuls

William B. West

John Rainsford

W. L. Barrington

John Shaw

Archibald McKenzie

Stephen M. MacKenzie

Arthur Donn Piatt

$1790-3$
$1794-1809$
$1809-26$
('pro tem')
$1826-47$
$1847-50$
$1850-3$
$1853-4$
$1853-4$ (agent)
$1854-5$
$1854-5$
$1855-8$
$1855-8$
$1859-61$
$1861-2$
$1869-71$
$1872-6$
$1876-81$
$1885-9$
$1889-93$
$1890-3$ (deputy)
1893
$1893-8$
$1898-1902$
$1902-4$
$1904-9$
$1909-19$

1863-7 (vice consul)

1871

$1871-2$

$1872-84$

$1884-6$

1886-93

1893 
Londonderry/Derry (consulate/consular agency)

Thomas Davenport

Thomas Harvey

James Corscaden

James McHenry

James Cairns

James McDowell

Robert Loughead

James Corscaden

James R. Smith, Jr.

James Corscaden

Alexander Henderson

James Corscaden

Thomas McGunn

Alexander Henderson

James Corscaden

Charles Dougherty

Felix Agmis

Robert C. Mack

D. H. Batchelder

Arthur Livermore

P. T. Rodger

Philip O'Hagan

Athlone (consular agency)

John Burgess

P. B. Treacy

Ballina (consular agency)

Robert Adam Kerr

Ballymena (consular agency)

William Young

Francis Skelly

George Ballentine

Wilson McKeown
1830-3 (vice consul)

1833-44 (vice consul)

1834-42

1842-5

1844-55 (vice consul)

1845 (declined appointment)

1845-54

1853-62 (agent)

1856-8

1857-8 (agent)

1858-61

1861-2

1861 (refused exequatur)

1862-3

1863-71 (vice consul)

1866 (not confirmed by Senate)

1867 (refused appointment)

1869 (resigned)

1870 (resigned)

$1870-86$

1888-c. 1905 (agent)

1908-20 (agent)

1888-1906

(interim)

$1900-2$

1843-5 (vice consul)

1845 to unknown (vice consul)

1871-1901 (agent)

1901-08 (agent) 
Crookhaven (consular agency)

Isaac Arthur unknown to 1862

Galway (consulate/consular agency)

Thomas M. Persse

$1832-44$

Michael Kennedy

$1844-7$

Thomas M. Persse

$1847-50$

John Duffy

$1853-5$

Valentine Hilmes

1855

Samuel W. Talbott

1856-9

Thomas M. Persse

1860-1

William B. West

1861-9

Robert A. Tennant

1901-38 (agent)

Kingstown (Dùn Laoghaire) (consular agency)

Michael Murphy

1857-unknown

Limerick (consular agency)

Michael Robert Ryan

1843-68, 1873-d. 1874

John Richard Tinsly

1874-90

Richard Hogan

1890-d. 1894

George P Mackenzie

1894

Edward Ludlow acting 1895

George Paul Mackenzie

1896-September 1896

Edward Ludlow

1896-1906

Lurgan (consular agency)

Frederick W. Magahan 1882-1908

Newry
Alexander F. Little
1842-4 (vice consul)
William R. Glenny
1844-8 (vice consul)
Charles Day
1848-52 (vice consul)
I. E. Carraher
1861-unknown (vice consul) 
Sligo (consular agency)

James Harper

William J. Eccles

John Tighe

Waterford (consular agency)

Thomas Evans

Benjamin Moore

William Farrell

Wexford (consular agency)

Francis Harper

Thomas Rowntree

Jaspar Walsh
1847-71 (vice consul)

1871-84

1884-90
1835

1864-d. 1879

1880-98

1832-59

1861-8

1868-unknown 


\section{Appendix 4.I}

\section{Table of prisoners claiming to be American citizens, 20 April I 866 (following September 1865 round-up)}

\begin{tabular}{clc}
\hline Number & Name & Prison \\
\hline 1. & Denis F. Burke & Mountjoy prison \\
2 & Michael Kerwin & ditto \\
3. & Bernard McDermott & ditto \\
4. & Daniel J. Mykins & ditto \\
5. & James Smith & ditto \\
6. & Frank Leslie (alias & ditto \\
& Eugene O'Shea) & \\
7. & Edward Maguigan & ditto \\
8. & James Burns & ditto \\
9. & Michael Duffy & ditto \\
10. & James Bible & ditto \\
11. & Eneas Doherty & ditto \\
12. & Maurice Fitzharris & ditto \\
13. & J. H. O'Brien & ditto \\
14. & Joseph P. Clery & ditto \\
15. & Thomas M. Costello & ditto \\
16. & J. O'Carroll & ditto \\
17. & Andrew J. Byrne & ditto \\
18. & William McGrath & ditto \\
19. & Hugh Dennedy & ditto \\
20. & Francis N. Kavanagh & ditto \\
21. & Edward Morley & ditto \\
22. & William Makay & ditto \\
23. & Patrick J. Condon & ditto \\
24. & O'Gorman Barry & ditto \\
25. & John Dunne & ditto \\
26. & John Sullivan & ditto \\
27. & James Murphy & ditto \\
28. & James Murphy & ditto \\
29. & John W. Byron & ditto \\
\hline & &
\end{tabular}




\begin{tabular}{|c|c|c|}
\hline Number & Name & Prison \\
\hline 30. & John H. Gleeson & Nenagh prison \\
\hline 31. & Joseph Gleeson & ditto \\
\hline 32. & William Quade & Mountjoy prison $^{1}$ \\
\hline 33. & Joseph H. Lawler & ditto \\
\hline 34. & James McDermott & ditto \\
\hline 35. & James Smith & ditto \\
\hline 36. & John A. Comerford & ditto \\
\hline 37. & Daniel C. Moynihan & ditto \\
\hline 38. & James E. McDermott & ditto \\
\hline 39. & George Smith & ditto \\
\hline 40. & Thomas J. Hynes & ditto \\
\hline 41. & Corporal McKay & ditto \\
\hline 42. & Thomas Hynes & ditto \\
\hline 43. & Michael O'Boyle & Kilmainhaim gaol $^{12}$ \\
\hline 44. & John Horan & ditto \\
\hline 45. & Thomas M. Holden & ditto \\
\hline 46. & Michael Fay & ditto \\
\hline 47. & M. F. Garvin M.D. & ditto \\
\hline 48. & Patrick Hart & Sligo gaol ${ }^{2}$ \\
\hline 49. & Michael McLoughlin & ditto \\
\hline 50. & James O’Byrne & Wicklow gaol ${ }^{2}$ \\
\hline 51. & Michael O’Brien & Tipperary bridewell \\
\hline 52. & Stephen F. Farrell & Roscommon gaol ${ }^{2}$ \\
\hline 53. & William Smith & Clonmel gaol $^{2}$ \\
\hline 54. & George Archdeacon & Mountjoy prison \\
\hline
\end{tabular}

* N.B. All the above may not have been and probably were not in prison on 20 April 1866 , the names of about ten were subsequently added by West.

1 Mountjoy prison, Kilmainhaim gaol were located in Dublin city.

2 'Gaol' is a historical spelling of 'jail'.

Source: NARA, S/D, Despatches from US consuls in Dublin, 1790-1906, vol. 6, roll 6, T199, Second series, enclosure in Larcom to West, 20 April 1866. 